\title{
Premiers résultats expérimentaux sur le comportement de Tabebuia heterophylla (DC) Britton et de Hymenaea courbaril L. vis-à-vis de la sécheresse
}

\author{
R. HUC \\ I.N.R.A., Station de Recherches forestieres \\ Centre de Recherches des Antilles et de la Guyane, Domaine Duclos, 97170 Petit-Bourg
}

\begin{abstract}
Résumé
Deux espèces de la forêt de basse altitude d'Amérique tropicale (Antilles), Tabebuia heterophylla (poiricr-pays) et Hymenaea courbaril (courbaril), se révèlent intéressantes pour le reboisement des régions les moins arrosées. Le comportement de ces espèces vis-à-vis de la sécheresse est étudié grâce à un traitement sous forme d'arrosage différencié appliqué à des jeunes plants âgés de 1 an, en pépinière.

L'évolution de l'état hydrique des plants est suivie au cours de 6 mois d'expérience à l'aide de la bombe à pression. Les valeurs caractéristiques du potentiel hydrique, du potentiel de base $(\mathrm{Pb})$ et du potentiel minimum $(\mathrm{Pm})$, sont étudiées. La cinétique journalière du potenticl de base indique que le poirier-pays se comporte comme une espèce plus économe de son eau que le courbaril. Toutefois, le meilleur enracinement du poirier-pays provoque un rapide dessèchement du sol. Ceci se traduit par une chute plus précoce du potentiel dc base de cette espèce dans les lots moins arrosés (arrosage 1 fois par semaine).
\end{abstract}

Durant la phase de dessèchement, l'évolution de la différence $\mathrm{Pm}-\mathrm{Pb}$ indique une fermeture stomatique maximale pour un potentiel de base plus élevé pour le poirier-pays ( -25 bars) que pour le courbaril ( -32 bars).

Les résultats obtenus dans les mesures d'élongation des axes montrent que l'arrêt de croissance pour les deux espèces se produit pour des valeurs élevées de $\mathrm{Pb}$ voisines de -6 bars pour le poirier-pays et -7 bars pour le courbaril.

Dans les deux cas, seule la fréquence de 1 arrosage hebdomadaire réduit de façon notable la formation de matière sèche et la croissance chez les plants.

\section{Introduction}

La revalorisation des sols impropres à l'agriculture et la reforestation des zones réputées «sèches» des Antilles rendent nécessaire la plantation d'essences forestières adaptées. Parmi les arbres pouvant convenir à cet usage, deux espèces ont plus particulièrement retenu l'attention des services forestiers à cause de leur utilisation traditionnelle en ébénisterie et du domaine climatique où elles se rencontrent : Tabebuia heterophylla (Bignoniaceae) et Hymenaea courbaril (Caesalpiniaceae). 
Tabebuia heterophylla (ou Poirier-pays) est une espèce d'Amćrique tropicale, qui recouvre l'ensemble de la forêt mésophile et la forêt xérophile (LASSERre, 1961). Elle atteint son meilleur développement à des altitudes basses en forêt mésophile. Elle se présente sous deux types morphologiquement distincts en Dominique, Martinique et Guadeloupe :

- Une variété à feuille simple que l'on rencontre dans les zones les plus arrosées au pied des massifs montagneux, exposés aux vents dominants.

- Une variété à feuille composée présente dans toutes les autres régions basses volcaniques ou calcaires. C'est cette variété à laquelle nous nous intéressons dans le présent travail.

Hymenaea courbaril (ou Courbaril) a une aire de répartition qui correspond à l'Amérique tropicale. Il se limite à la forêt mésophile où il se cantonne à des altitudes basses $<400 \mathrm{~m}$ ct dans des régions à précipitations modérées comprises entre 500 et $2000 \mathrm{~mm} / \mathrm{an}$. Toutefois, à la différence des «poirier-pays », il a une nette préférence pour des sols volcaniques.

Les travaux concernant la résistance à la sécheresse chez ces deux espèces sont quasi-inexistants. Les relations entre le dessèchement du sol et la phénologie ont été étudiées chez une autre espèce du genre Tabebuia ( $T$. neochrysantha) par REICH \& Borchert (1982). Toutefois, l'évolution de l'état hydrique des plantes au cours du dessèchement n'est pas abordée par ces auteurs.

Il nous a alors paru intéressant de préciser par un traitement expérimental le tempérament de ces deux espèces vis-à-vis de la sécheresse du sol.

\section{TABLEAU 1}

Caractéristiques générales des espèces.

General characteristics of the species.

\begin{tabular}{|c|c|c|}
\hline & Tabebuia heterophylla & Hymenaea courbaril \\
\hline Type de forêt & mésophile et xérophile & mésophile \\
\hline Sociabilité & grégaire & dispcrsée, çà et là \\
\hline Agressivité & colonisatrice de zones dégradées & faible pouvoir de colonisation \\
\hline Importance relative .... & très répandue & rare; espèce menacée \\
\hline Régénération $\ldots \ldots \ldots$. & abondante & faible \\
\hline Usages & $\begin{array}{l}\text { ébénisterie (bois clair de faible } \\
\text { densité) }\end{array}$ & $\begin{array}{l}\text { ébénisterie (bois brun de forte } \\
\text { densité) }\end{array}$ \\
\hline Sols & calcaire ou volcanique & $\begin{array}{l}\text { presque exclusivement volcani- } \\
\text { que }\end{array}$ \\
\hline Modèle architectural & Koriba & Troll \\
\hline
\end{tabular}




\section{Matériel et méthodes}

Afin de comprendre le comportement vis-à-vis de la sécheresse de ces deux espèces, nous leur avons fait subir des traitements où l'on contrôle l'apport d'eau aux plants. Trois dispositifs ont été mis en place en trois localités de Guadcloupe afin de répéter les mêmes traitements en des endroits où les conditions atmosphériques générales sont sensiblement différentes (cf. tabl. 2).

\section{TABLeaU 2}

Quelques données climatiques sur les trois stations choisies.

Mois à fortes précipitations (septembre 82) et mois à faibles précipitations (février 83) (Bulletin Climatologique Mensuel de la Commission Météorologique Départementale de Guadeloupe).

Some climatic data on the locations of the study - very rainy month (september 82) and very dry month (february 83) (Monthly Weather Report of the Meteorological Board of the Department of Guadeloupe).

\begin{tabular}{|c|c|c|c|c|c|c|c|c|c|c|}
\hline & \multirow{2}{*}{\multicolumn{2}{|c|}{$\begin{array}{l}\text { Pluviométrie } \\
\text { mensuelle }\end{array}$}} & \multicolumn{4}{|c|}{ Humidité relative } & \multicolumn{4}{|c|}{ Température moyenne } \\
\hline & & & \multicolumn{2}{|c|}{ sept. 82} & \multicolumn{2}{|c|}{ fév. 83} & \multicolumn{2}{|c|}{ sept. 82} & \multicolumn{2}{|c|}{ fév. 83} \\
\hline & sept. 82 & fév. 83 & $\operatorname{Max}$ & Min & $\operatorname{Max}$ & Min & $\operatorname{Max}$ & $\operatorname{Min}$ & $\operatorname{Max}$ & Min \\
\hline Abymes & 233,0 & 8,4 & $31^{\circ} 3$ & $23^{\circ} 1$ & $29^{\circ} 1$ & $19^{\circ} 8$ & $97 \%$ & $60 \%$ & $97 \%$ & $53 \%$ \\
\hline Basse-Terre & 196,5 & 12,3 & $30^{\circ} 4$ & $23^{\circ} 3$ & * & * & $95 \%$ & $66 \%$ & * & * \\
\hline Duclos .. & 437,1 & 52,9 & $29^{\circ} 1$ & $22^{\circ} 0$ & $26^{\circ} 6$ & $19^{\circ} 1$ & $96 \%$ & $78 \%$ & $97 \%$ & $74 \%$ \\
\hline
\end{tabular}

* Données manquantes.

Pour les pépinières de Pointe-à-Pître et de Blanchet, les données sont celles du poste le plus proche, respectivement Abymes et Basse-Terre.

Les deux espèces ont été prélevées à l'état de semis ayant entre 15 et $20 \mathrm{~cm}$ environ, puis repiquées en pots de 8 litres et élevées en pépinière.

Pour le «poirier-pays», les plants proviennent d'une même localité de Guadeloupe, Dévarieux, Morne-à-l'cau. Il s’agit de la variété à feuilles composées, représentée aussi sur l'ensemble de la Grande-Terre. En ce qui concerne le courbaril, les plants sont issus de graines de peuplement naturel de l'île des Saintes, Terre-de-Bas, Guadeloupe, élevés au préalable à la pépinière de la Providence (Petit-Bourg, Guadeloupe) par l'Office National des Forêts.

Le protocole expérimental adopté pour les deux espèces est le même dans chaque dispositif.

L'ensemble du dispositif est recouvert d'un film plastique transparent ouvert sur les côtés pour permettre une bonne aération.

Afin d'éviter les biais expérimentaux dûs aux effets de bordure (pluie apportée par le vent), les pots subissent une rotation hebdomadaire. 
Nous disposons de 100 plants par espèce et par dispositif qui sont arrosés régulièrement durant 2 mois pour permettre une bonne reprise, puis répartis en trois lots soit :

$1^{* r}$ lot, 33 plants, arrosés 6 fois par semaine $(6 \times)$.

$2^{*}$ lot, 33 plants, arrosés 3 fois par semaine $(3 \times)$.

3 * lot, 34 plants, arrosés 1 fois par semaine $(1 \times)$.

A chaque arrosage, les plants reçoivent une dose d'eau équivalente à $600 \mathrm{ml}$, suffisante pour ramener le sol des lots $1 \times$ et $3 \times$ à une humidité pondérale voisine de celle du lot $6 \times$; soit respectivement 15 p. 100 et 18 p. 100 , contre 17 p. 100 atteint par le lot $6 \times$ après ressuyage complet.

Le comportement des plants est suivi durant 8 mois (octobre 1982 à juin 1983) pour la croissance et 6 mois (décembre 1982 à juin 1983) pour l'état hydrique. Différentes opérations ont été effectuées :

- Mesure des pF du sol et établissement des relations entre humidité pondérale du sol et $\mathrm{pF}$, puis entre humidité pondérale du sol et potentiel hydrique des plants.

Les mesures d'humidité pondérale sont faites à partir d'une fraction correspondante à environ $1 / 3$ de la totalité du sol du pot, obtenue après homogénéisation. Cette opération a été placée en fin d'expérimentation.

- Mesure de croissance de la totalité du plant pour le poirier-pays et de 2 axes par plant pour le courbaril, tous les 15 jours.

- Mesure du potentiel hydrique ou de sève à l'aide de la bombe à pression (Scholander et al., 1965). Ces opérations de mesure sont effectuécs uniquement à la pépinière de Blanchet, sur des feuilles prélevées dans la même zone des plants, soit donc à partir de la $2^{\prime \prime}$ feuille complètement développée en-dessous de l'apex. Nous avons ainsi pu effectuer 3 types de mesures :

- Mesure du potentiel de fin de nuit ou potentiel de base (Pb) de 5 plants par lot, chaque semaine avant l'arrosage, puis le lendemain pour les lots $1 \times$ et $3 \times$, et uniquement le lendemain du $1^{\text {"r }}$ arrosage pour le lot $6 \times$.

- Mesure du potentiel hydrique au cours d'un cycle de 24 heures, afin de suivre l'évolution de ce potentiel en fonction de l'heure solaire.

- Mesures au cours d'un dessèchement sur 8 jours des $\mathrm{Pb}$ et $\mathrm{Pm}$ (potenticl minimum atteint dans la journée).

— Calcul du poids de matière sèche formée par les plants en fin de traitement.

Au cours du traitement, les lots secs ont été réhydratés, par arrosage de fréquence $6 \times$ durant 2 semaines à Blanchet et Pointe-à-Pître et 3 semaines à Duclos, ce quii permet de suivre la croissance en fonction du changement d'alimentation hydrique. 


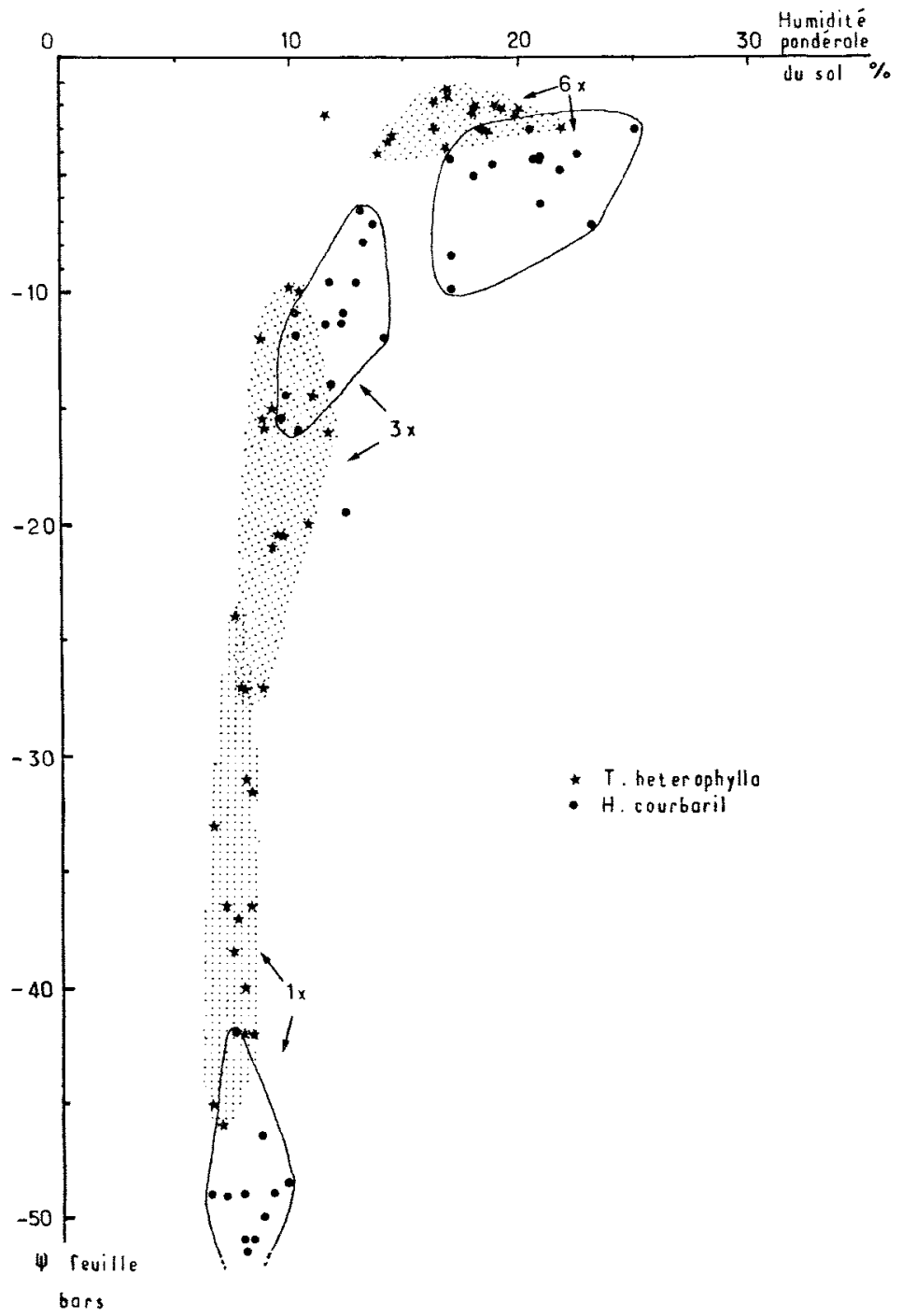

FIG. 1

Distribution des potentiels hydriques de base (Pb) des plants pour Tabebuia heterophylla et Hymcnaca courbaril, en fonction de l'humidité pondérale du sol et des traitements [arrosage 6 fois $(6 x) ; 3$ fois $(3 x)$ et 1 fois (1x) par semaine].

The relationship between predawn potential and soil water content in Tabebuia heterophylla and Hymenaea courbaril seedlings within the different watering regimes [watering 6 times $(6 x) ; 3$ times $(3 x)$ and once $(1 x)$ a week]. 


\section{Résultats}

\subsection{Hétérogénéité des lots et relation entre potentiel de base et humidité du sol}

La figure 1 montre la relation liant le potentiel de base des plants et l'humidité pondérale. Nous retiendrons les faits suivants :

- Il existe à l'intérieur d'un même traitement une grande dispersion des valeurs de $\mathrm{Pb}$. Cette dispersion est importante lorsque les lots deviennent plus secs. Dans le lot sec de courbaril $(1 \times)$, certains plants ont un $\mathrm{Pb}$ qui atteint la limite de la pression mesurable dans la chambre. La variabilité du $\mathrm{Pb}$ à l'intérieur des traitements tient à la variabilité entre les plants qui épuisent, par un réseau racinaire ou unc surface foliaire plus ou moins importante, de façon variable, les réserves hydriques du sol. On notera par ailleurs que, pour des humidités pondérales voisines de 10 p. 100 et en-dessous, de faibles variations d'humidité du sol provoquent des écarts importants de $\mathrm{pF}$ donc de $\mathrm{Pb}$.

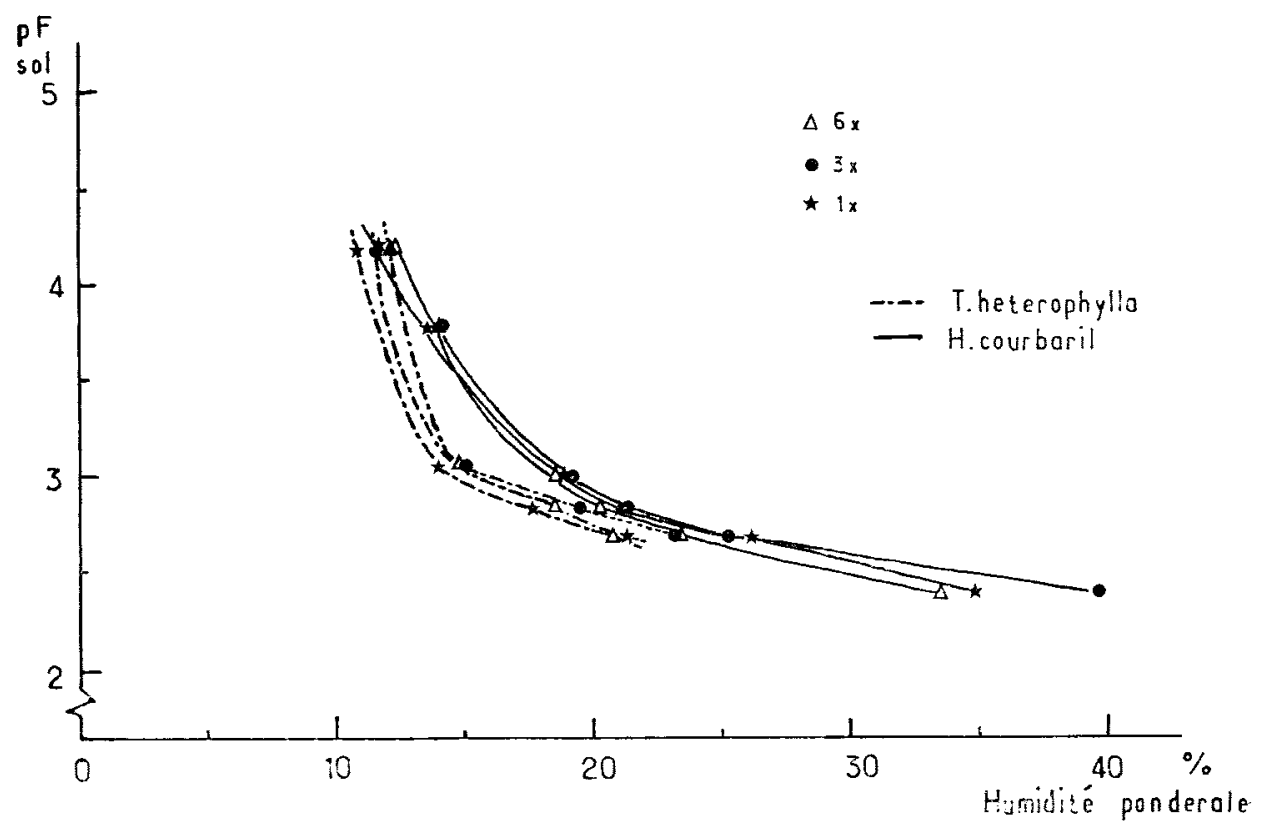

FIG. 2

Relation entre le $p F$ du sol et l'humidité pondérale dans les différents lots de Tabebuia heterophylla et Hymenaea courbaril.

The relationship between $p F$ and soil water content within the different watering regimes applied to Tabebuia heterophylla and Hymenaea courbaril seedlings.

- Chez les plants de courbaril, on observe, pour un potentiel de base équivalent aux plants de poirier-pays correspondants, une humidité pondérale du sol plus impor- 
tante. Ceci peut être dû au fait que le col du poirier-pays tend à se dessécher plus facilement que celui du courbaril. La courbe $\mathrm{pF}$ en fonction de l'humidité pondérale ciu sol confirme la nature plus sableuse du sol des plants de poiricr-pays (fig. 2). Cette distorsion est provoquée par lintroduction au moment de la plantation du courbaril d'une certaine quantité de terre argileuse présente autour des racines. Cependant, même en tenant compte de ce phenomène, l'écart entre les réponses des deux cspèces subsistc. Ceci nous conduit à envisager une explication au niveau racinaire. La mesure du $\mathrm{Pb}$ correspond à l'état d'humidité du sol voisin des racines. Or si les racines ne colonisent qu'une partie du sol, cette fraction est, par suite de la résistance au transfert d"eau dans le sol, dans un état hydrique plus faible que l'ensemble du sol du pot. Le fait que l'enracinement soit moins développé chez le courbaril que chez. le poirier-pays entrânerait un $\mathrm{Pb}$ plus faible à teneur en eau égale ou à $\mathrm{Pb}$ égal des teneurs en eau plus fortes. Ce que lon sait du développement racinaire du poirierpays et du courbaril dans leur milieu naturel nous confirme dans cette hypothc̀se que le poirier-pays est mieux adapté pour l'exploration du milieu par les racines.

\subsection{Evolution quotidienne du potentiel de sève}

Les courbes obtenues (fig. 3) montrent que le potentiel descend progressivement à partir de $6 \mathrm{~h}$ (temps solaire moyen), pour atteindre un minimum vers $14 \mathrm{~h}$. Le retour aux potentiels élevés s'effectue en un temps deux fois moindre. L'équilibre final étant atteint vers $24 \mathrm{~h}$.

Les courbes obtenues dans les lots sees (fig. $3 \mathrm{C}$ et $\mathrm{D}$ ) indiquent une grande dispersion des valeurs de potentiel due au fait que l'on opère sur des ensembles de plants différents (chaque point correspond à la moyenne de 5 plants par lot). On notera aussi labsence de l'état d'équilibre puisque l'on atteint le 18/06 un potentiel de base plus faible que le $\mathrm{Pb}$ du matin du 17/06. On observe également une redescente du potentiel au cours de la nuit. Cette évolution inhabituelle peut s'expliquer par laction d'un vent fort, persistant depuis $19 \mathrm{~h}$ le $17 / 06$.

L.a comparaison des lots humides (fig. $3 \mathrm{~A}$ et $\mathrm{B}$ ) montre une différence de réponsc des deux espèces. Si le $\mathrm{Pb}$ est sensiblement le même (voisin de -4 bars), le potentiel minimum (ou Pm) est plus bas chez le courbaril. La différence entre le $\mathrm{Pm}$ et le $\mathrm{Pb}$ est de l'ordre de -8 bars pour le poirier-pays et -16 bars pour le courbaril. Ceci peut être provoqué, toutes choses égates par ailleurs, par une plus forte résistance au transfert d'eau du sol vers la plante dû au rapide épuisement en eau de la zone immédiatement utilisée par le réseau racinaire, ou bien à une résistance au transfert de l'eau des racines vers les feuilles plus grande chez le courbaril, à transpiration égale, ou encore dû, pour une résistance au transfert équivalente, à unc plus forte transpiration du courbaril (régulation stomatique moins efficace). Si l'or. admet l'hypothèse d'une disponibilité en eau équivalente dans la zone racinaire (valable pour des plants bien hydratés), on est ramené aux deux dernières hypothèses indiquant que le poirier-pays serait une espèce plus économe de son eau.

Pour les lots à sol plus desséché (arrosage $1 \times$ ), les écarts $\mathrm{Pm}-\mathrm{Pb}$ sont sensiblement les mêmes (-12 bars); par contre on retrouve des plants de courbaril globalement à un état hydrique plus faible que ceux du poirier-pays, confirmant une moins bonne disponibilité en eau du sol à tout moment de la journée. 

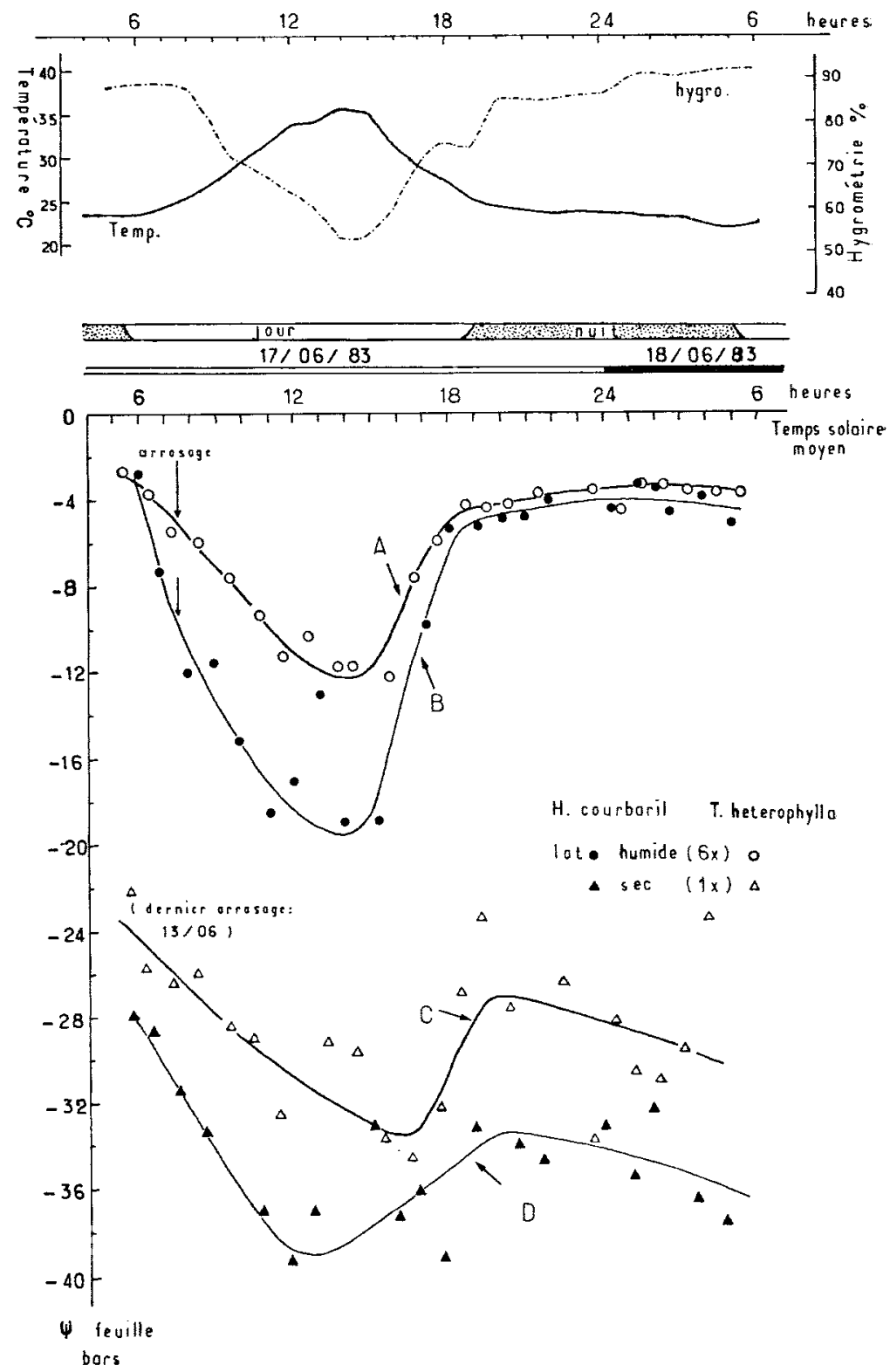

FIG. 3

Cinétique journalière du potentiel hydrique des plants de Tabebuia heterophylla et Hymenaea courbaril, pour des lots bien arrosés (6x) ( $A$ et $B)$ et des lots secs $(1 x)(C$ et $D)$. Température et hygrométrie enregistrées au cours de la même période.

Diurnal change in water potential in Tabebuia heterophylla and Hymenaea courbaril seedlings growing on humid $(6 x)$ ( $A$ and $B$ ) and dry soils $(1 x)(C$ and $D)$.

Temperature and hygrometry recorded at the same time. 


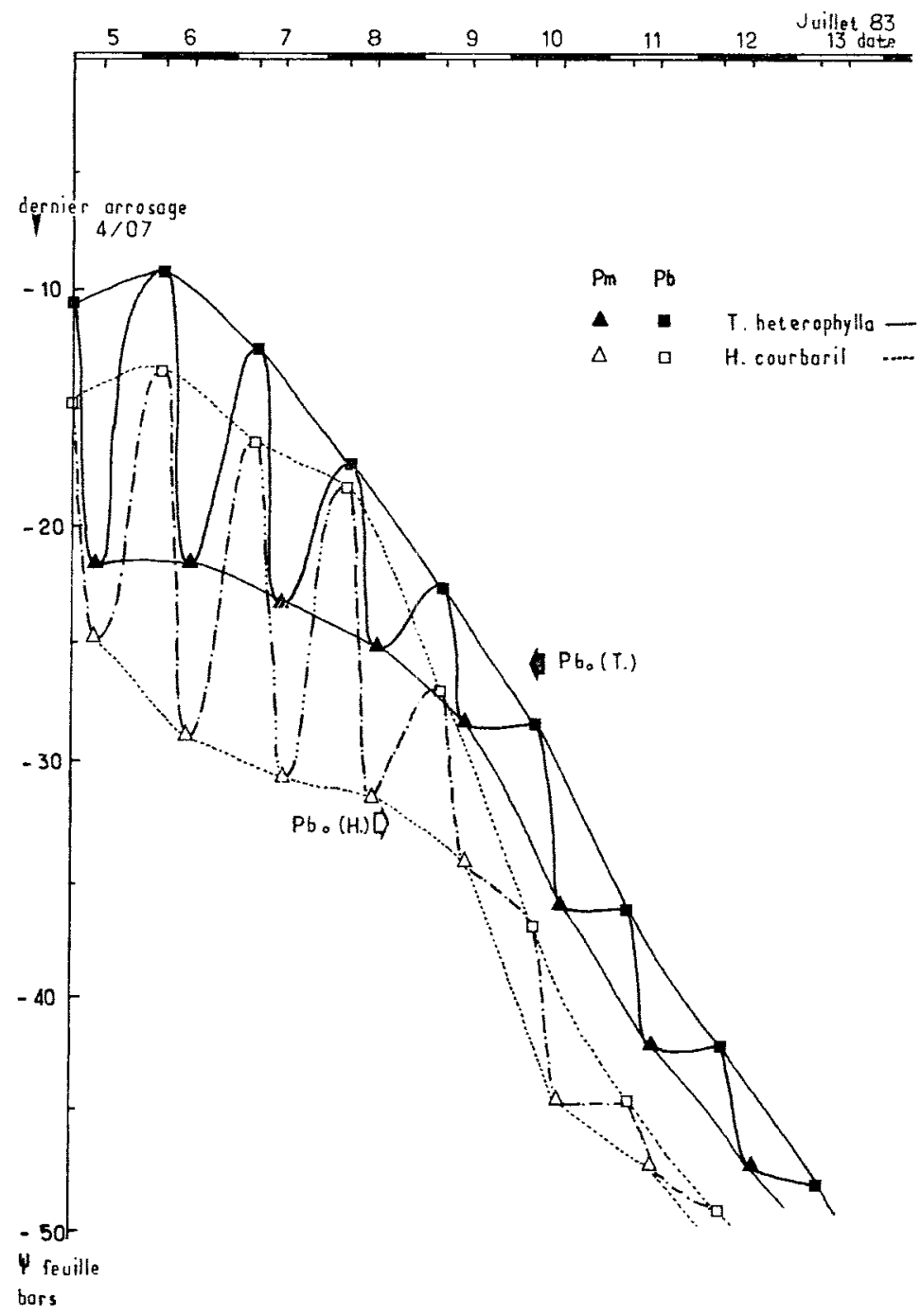

FIG. 4

Evolution du potentiel hydrique (potentiel de base $\mathrm{Pb}$, et potentiel minimum Pm) au cours du dessèchement de jeunes plants de Tabebuia heterophylla et Hymenaea courbaril : les flèches indiquent le potentiel de base critique $P b_{o}$.

Evolution of the water potential (predawn potential $P b$, and minimum potential $P m$ ) in Tabebuia heterophylla and Hymenaea courbaril seedlings during the course of soil drying; arrows indicate critical predawn potential $P b_{0}$. 


\subsection{Cinsitique du dessèchement}

Elle est obtenue en suivant chaque jour le petentiel de case et le potentiel minimum atteints par un ensemble de 5 plants de poirier-pays et 5 plants de courbaril en l'absence d’arrosage. Les courbes représentées sur la figure 4 montrent lévolution du potentiel hydrique chez des plants du lot $1 \times$ arrosés la veille (4/06). La valeur du potenticl de base le $5 / 06$ - 15 bars pour le courbaril; -10.5 bars pour le poirier-pays - indique que les plants nont pas subi une réhydratation complète. Au cours du dessèchement, on constate un rapprochement des valeurs des $\mathrm{Pb}$ et $\mathrm{Pm}$ qui s'obtient sensiblement après 6 jours. Il y a done une fermeture progressive des stomates afin de limiter les pertes deau par transpiration, ce qui se traduit par la diminution des fluctuations journalières jusquà obtenir un écart $\mathrm{Pm}-\mathrm{Pb}$ plus au moins constant à partir d'un certain potentiel que l'on qualifiera, suivant Aussenac \& Granier (1978), de potentiel critique $\left(\mathrm{Pb}_{6}\right) ; \mathrm{Pb}_{0}$ peut être considéré comme une caractéristique des espèces. On obtient un $\mathrm{Pb}_{11}$ proche de -25 bars pour Tabebuia heterophylla et -32 bars pour $H$. courbaril. Cependant, le dessèchement est ici très rapide et écrase le phínomène. A titre de comparaison, on peut citer les valeurs trouvées pour le Douglas (Pseudcitsuga menziesii) par Waring \& Cleary (1967), - 25 bars, ou par Aussenac \& Granier (1978) pour Picea abies ( -20 bars).

On peut également noter que la régulation des pertes d'eau est différente selon les espèces. Chez Ie poirier-pays, à partir du point le plus élevé de $\mathrm{Pb}$, la diminution du potenticl hydrique est plus rapide que chez le courbaril, les deix $\mathrm{Pb}$ se rejoignant presque vers -18 bars. Cependant lamplitude reste élevée plus longtemps pour le courbaril alors qu'elle diminue progressivement chez le poirier-pays. Ceci pourrait traduire le fait que le poirier-pays commence très tôt à épuiser l'eau de son milieu (meilleur enracinement), mais le phénomène s'inverse par la suite car l'enveloppe $\mathrm{Pm}-\mathrm{Pb}$ s'effondre sinon plus tôt, du moins plus rapidement chez le courbaril, indiquant un rapide dessèchement du sol. Il serait intéressant de mettre en relation les valeurs de $\mathrm{Pb}$, obtenues avec le $\mathrm{Pb}$ à partir duquel les fonctions photosynthétiques s'annulent.

Les valeurs minimales de $\mathrm{Pb}$ enregistrées au cours du dessèchement, de l'ordre de -50 bars, sont atteintes très rapidement. Nous avons été limités par les possibilités de la chambre à pression clle-même. Malgré ces valeurs très basses de $\mathrm{Pb}$, un réarrosage des plants provoque un retour rapide à un $\mathrm{Pb}$ élevé sans aucun dommage pour les plants.

\subsection{Evolution au cours de l'année du potentiel de base et de la croissance en fonction des traitements}

\section{- Effet des différentes fréquences d'arrosage sur l'évolution du potentiel de base}

Les cinétiques des potentiels de base sur 6 mois pour les deux espèces (fig. $9 \mathrm{~B}$ et $\mathrm{C}, 10 \mathrm{~B}$ et $\mathrm{C}$ ) sont établies à partir de mesures effectuées sur 5 plants par lot. Elles confirment le fait que l'écart entre les valeurs extrêmes du $\mathrm{Pb}$ est directement lić à l'arrosage (lots $3 \times$ et $\mid \times$ ). Cet écart reste en effet plus important dans le lot $1 \times$ par rapport à $3 \times$.

On notera que dans le cas du lot $3 \times$, l'évolution hebdomadairc du potentiel résulte d'une succession de 3 ondulations liées aux arrosages répétés. Nous en avons 
donné une représentation simplifiée en réunissant par une droite les points extrêmes de la semaine.

Il est important de noter que dani le cas des lots déshydratés on ne revient pas, ainsi que l'on pourrait s'y attendre, a 1 potentiel le plus élevé (voisin de -5 bars) lors du réarrosage. Il est probable que, à cause des phénomènes de retrait du sol provoqués par le dessèchement, une certaine partie de l'eau s'écoule hors du pot par des circuits privilégiés et la fraction restante est rapidement mobilisée pour l'évapotranspiration dans la journée qui suil. Les tissus des plants ne retrouvent pas un état de pleine turgescence; ils ne sonı que partiellement réhydratés.

On note par ailleurs que les amplitudes de variations sont plus importantes pour le poirier-pays que pour le courbaril (lots $3 \times$ et $1 \times$ ). Ceci conforte l'hypothèse d'un épuisement rapide des réserves hydricues par les plants de poirier-pays dû à une meilleure occupation du sol par leur système racinaire, et vraisemblablement aussi à une surface évapotranspirante un pelı plus importante. L'apparente contradiction de ces résultats avec ceux obtenus lors du dessèchement (fig. 4) tient au fait que dans l'expérimentation de dessèchement, le $\mathrm{Pb}$ des plantules de courbaril est après arrossage plus faible que celui du poirier-pays, mais il évolue moins vite. Cependant, lorsque l'épisode de dessèchement se prolongte et que les $\mathrm{Pb}$ atteignent les faibles valeurs -25 à -30 bars, il y a inversion du phénomène et l'état hydrique du courbaril évolue plus vite que celui du poirier-pàs. C'est ce qui est exprimé dans les figures 1 et 3 .

\section{- Influence des précipitations et de l'état hygrométrique de l'air sur le Pb}

Le type de couvert utilisé pour les dispositifs nous a permis d'éviter les effets directs de la pluie, mais les plants restent soumis à des aléas climatiques qui ont pour effet de limiter leur évapotranspiration : ciel couvert, gouttelettes d'eau fines déposées par le vent sur les feuilles et le sol lors des pluies, état hygrométrique élevé de lair.

Ainsi, la remontée des valeurs de $\mathrm{Pb}$ des lots $3 \times$ et $1 \times$ pour le poirier-pays peut être associée aux épisodes pluvieux de fin décembre-début janvier. Le phénomène est également sensible pour les lots correspondants de courbaril. De même, la remontée du potentiel du début du mois de mars serait une conséquence des pluies de cette période. Lorsque la demande évapo-transpiratoire de lair est faible, le dessèchement du sol est moindre, la réhydratation est plus poussée, et la recharge des plants meilleure. Inversement, les vents secs de la période de "carême " (février à avril) accélèrent le phénomène de dessèchement.

\section{- Effet de l'arrosage sur la croissance}

\section{a) Croissance globale}

Les résultats obtenus sont reproduits dans le tableau 3 et les courbes 5, 6, 9 et 10. Ainsi dans chaque dispositif et pendant une période d'arrosage différencié stricte, une croissance en longueur différente est obtenue suivant les lots. L'analyse de variance révèle que les résultats de croissance correspondant à la période d'arrosage différencié (cf. tabl. 3) sont significativement différents, pour les lots pris deux à deux ct à l'intéricur de chaque dispositif, uniquement entre le lot $1 \times$ et chacun des autres lots (seuil de probabilité : 0,5 p. 100). 


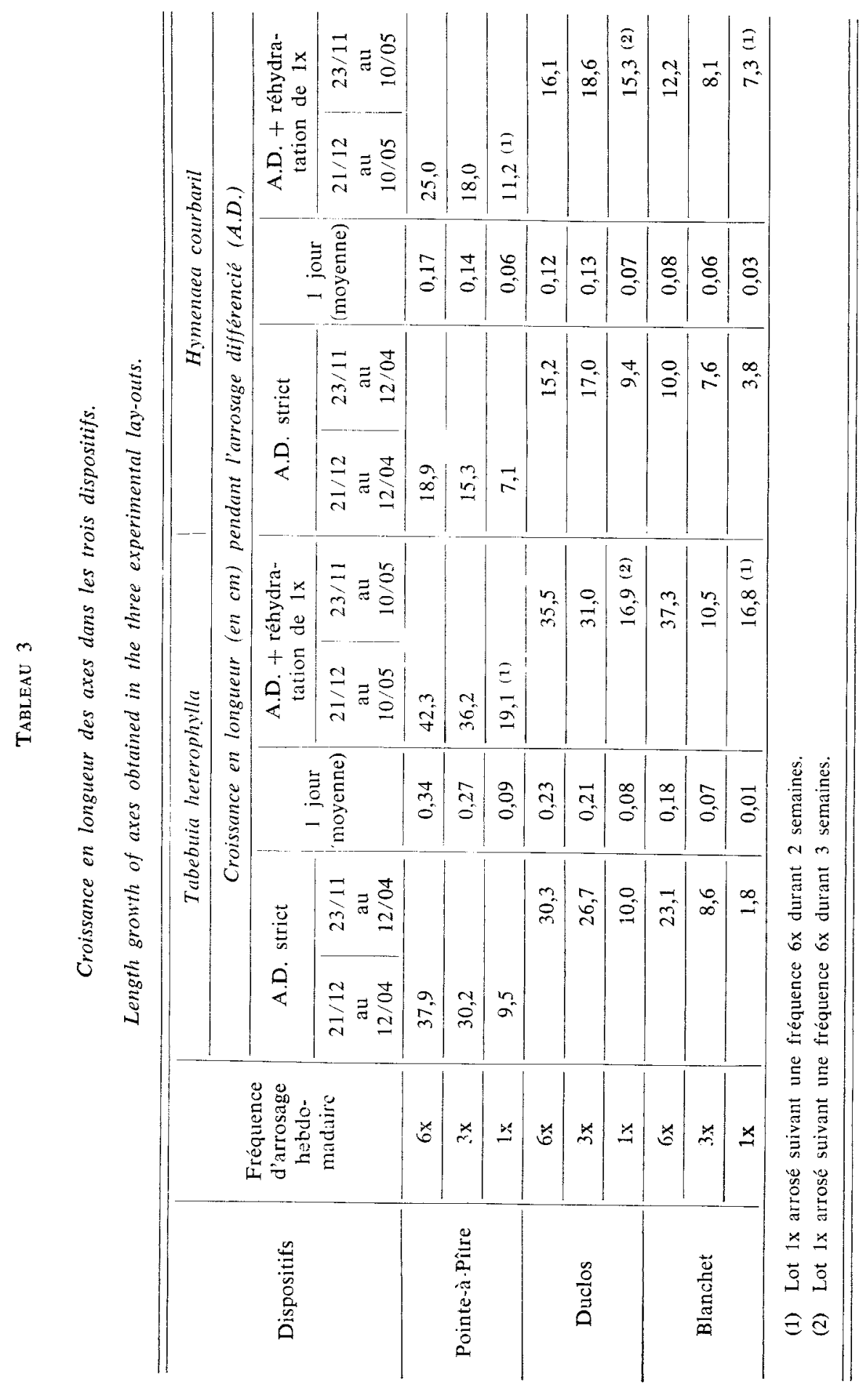



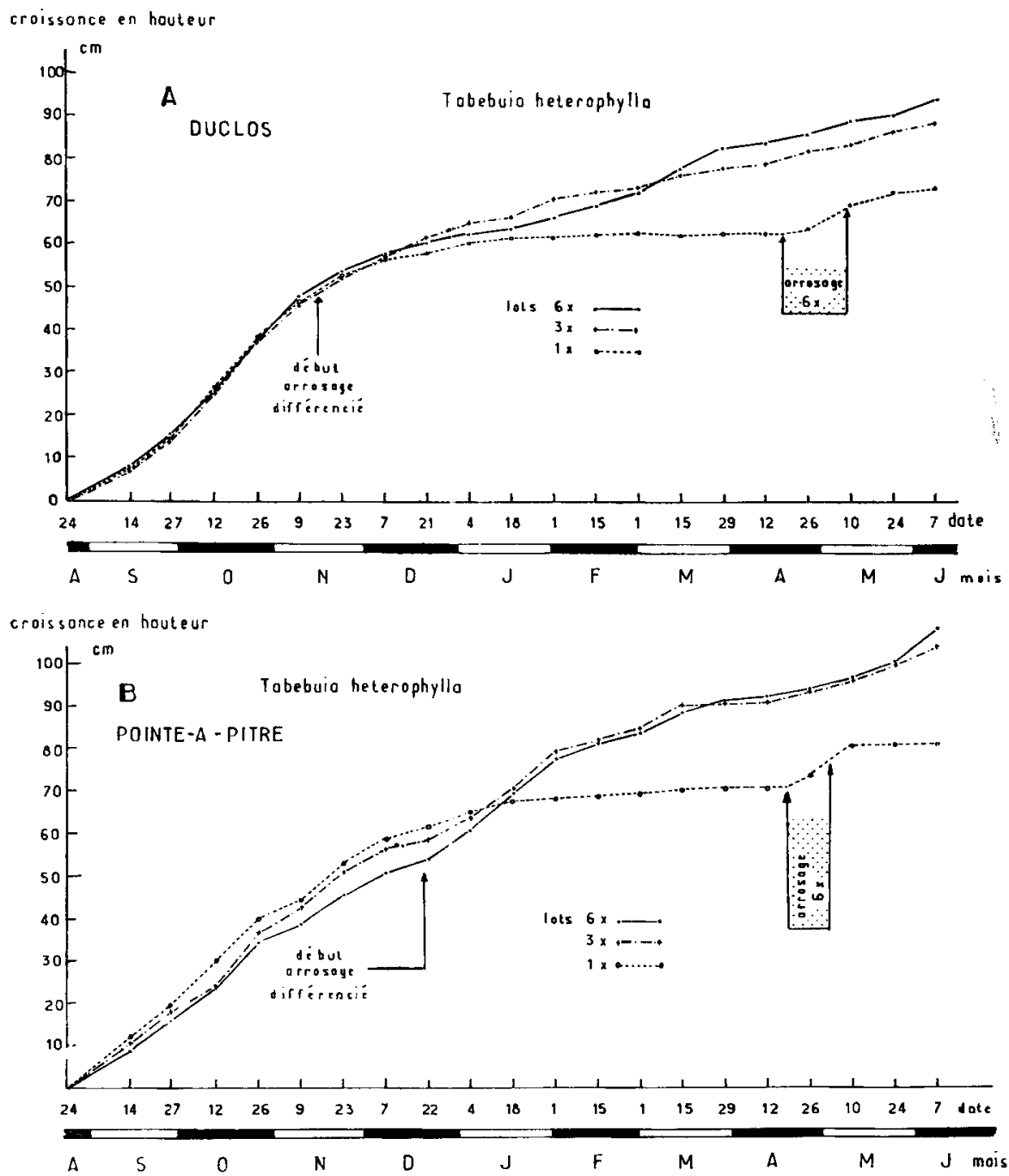

FIG. 5

Croissance en hauteur des plants de Tabebuia heterophylla dans les dispositifs de Duclos (A) et de Pointe-à-Pître $(B)$.

Height growth of Tabebuia heterophylla seedlings obtained at Duclos $(A)$ and Pointe-à-Pître (B) experimental lay-outs. 


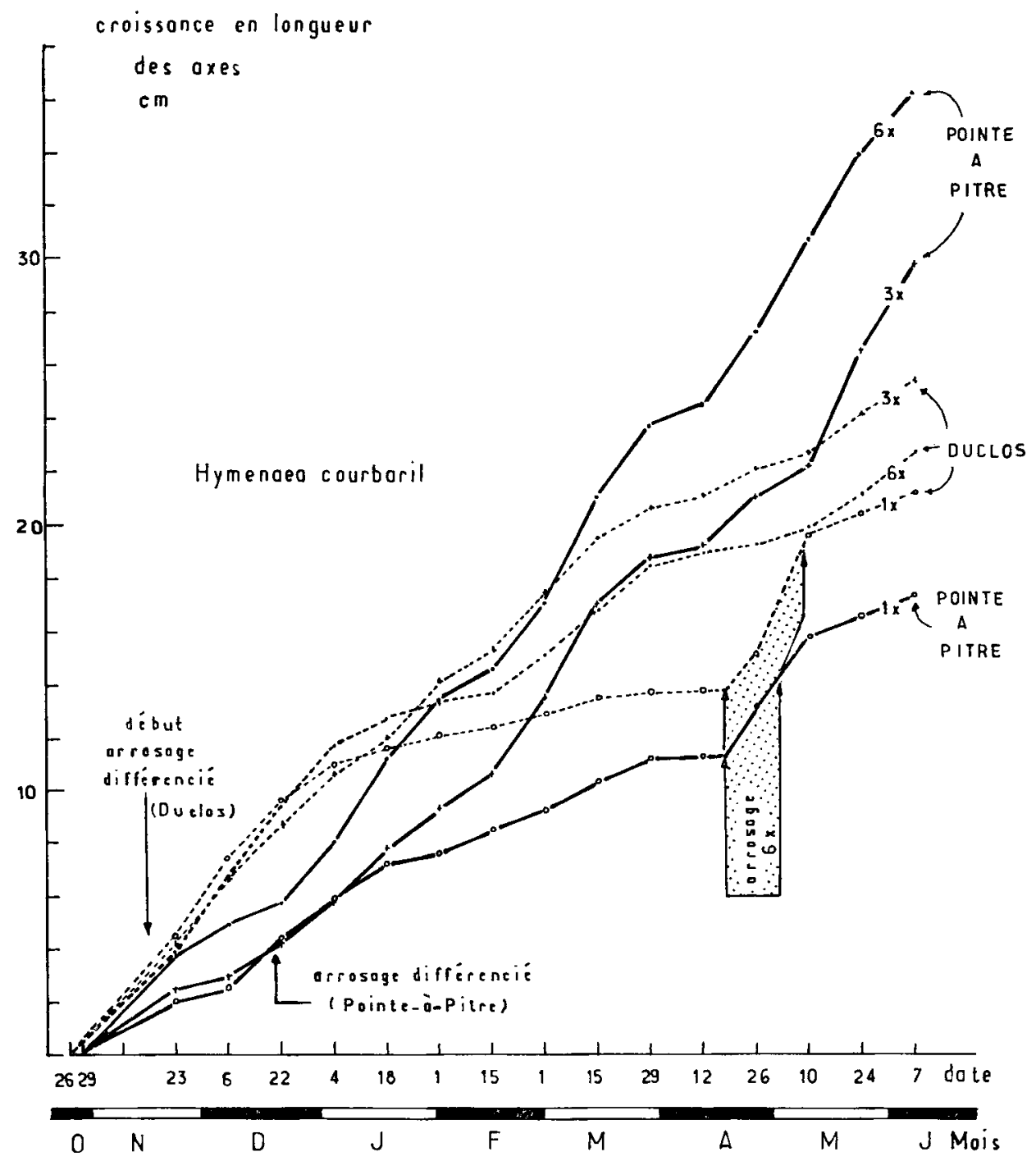

FIG. 6

Croissance en longueur des axes des plants de Hymenaea courbaril dans les dispositifs de Duclos et de Pointe-à-Pître.

Length growth of axes of Hymenaca courbaril seedlings at Duclos and Pointe-à-Pître experimental lay-outs. 
On notera que la différence de croissance entre les lots $3 \times$ et $6 \times$ est faible mais reste à l'avantage du lot $6 \times$ sauff pour le courbaril à Duclos (tabl. 3 et fig. 6) où le lot $3 \times$ manifeste une meilleure croissance. Dans tous les cas, la croissance cst la plus faible pour les plants du lot $1 \times$. Cette croissance représente environ 38 à 55 p. 100 de la croissance du lot le plus vigoureux pour le courbaril et entre 8 et 33 p. 100 de celle du lot le plus vigoureux correspondant pour le poirier-pays.

L'évolution au cours du temps de la croissance - fig. 5, 7 et $9 \mathrm{D}$ pour le poirierpays ; 6,8 et $10 \mathrm{D}$ pour le courbaril - montre que :

- l'effet de la réduction de l'arrosage sur la croissance ne se manifeste que lentement. Le temps de réaction est de lordre de 1 à 2 mois;

- il y a dans certains cas un arrêt total de la croissance lié à la persistance de l'arrosage réduit $(1 \times)$;

- lorsque le lot $1 \times$ est réhydraté par un arrosage $6 \times$, il y a un rattrapage partiel de la croissance. Ceci se produit pour le courbaril à Blanchet et à Duclos où les axes du lot $1 \times$ arrivent par cet arrosage à égaler respectivement les axes des lots $3 \times$ et $6 \times$. Pour le poirier-pays à Duclos, cet arrosage intensif permet aux plants du lot $1 \times$ de dépasser ceux du lot $3 \times$.

On remarque que les plants ont, quels que soient les lots, une meilleure croissance d'abord à Pointe-à-Pître, puis a Duclos; le dispositif de Blanchet étant celui où la croissance est la moins forte. On peut expliquer cette différence d'abord par un sol plus riche pour les pots de Pcinte-à-Pître et Duclos, et aussi par les prélèvements réguliers de feuilles pour les inesures de potentiel à Blanchet, qui réduisent dans une certaine mesure les surfaces assimilatrices. La meilleure croissance à Pointeà-Pître par rapport à Duclos peut s'expliquer par des conditions d'évapotranspiration moins rigoureuse dans la pépinière de Pointe-à-Pître dues à la présence d'arbres autour du dispositif.

\section{b) Croissance individuelle}

Les figures 7 et 8 représentent cuclques cas de croissance individuelle.

- Chez le poirier-pays (fig. 7), on n'observe pas à Duclos de rythme de croissance au cours de la période d'étude. Cependant à Blanchet (fig. 9 D), il y a une accélération sensible de la croissance de l'ensemble des plants des lots $3 \times$ et $6 \times$ en mai et juin que l'on peut mettre en relation avec une hygrométrie plus élevée de l'air à cette période.

La formation de ramifications, donnant naissance à chaque fois ̀̀ 1 relais du tronc et 1 branche (modèle de Koriba), n'a en apparence aucun effet notable sur la croissance. L'arrosage réduit $(1 \times$ ou $3 \times)$, en diminuant la croissance, ne fait que retarder la formation des ramifications. Il n'y a donc pas synchronisation de la ramification entre les plants des différents lots, ni même entre les plants du même lot. Ainsi, dans le lot $3 \times$ (fig. 7), le plant 57 qui a une meilleure croissance que le 97 se ramifie plus tôt.

- Chez le courbaril, les courbes de croissance individuelle établies à partir de quelques axes appartenant à 4 plants différents (fig. 8) permettent de montrer que : 


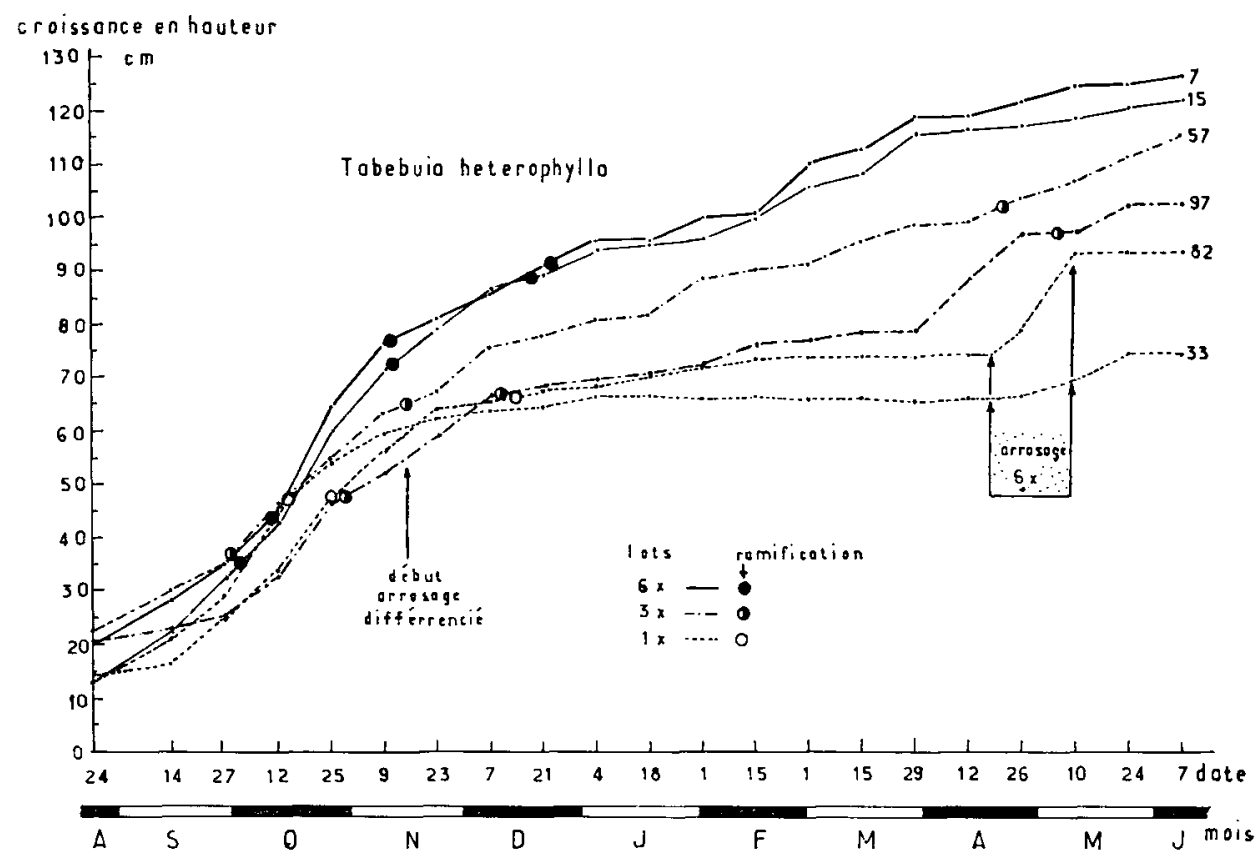

Frg. 7

Quelques exemples de croissance individuelle de plants de Tabebuia heterophylla dans le dispositif de Duclos.

Some examples of growth of individual plants of Tabebuia heterophylla at Duclos experimental lay-out.

- la croissance de chaque axe suit un certain rythme de repos et d'élongation;

- ce rythme de croissance est à peu près le même sur un même individu (exemple des axes a et b du plant 28) ; par contre, à l'intérieur d'un même lot, chaque individu suit son propre rythme. Ainsi, lorsque l'on s'adresse à un ensemble de plants, ce rythme de croissance individuelle s'estompe et tend à disparaître ;

- on notera par ailleurs que l'arrosage $1 \times$ n'annule pas complètement le rythme de croissance. Seule l'amplitude des poussées des axes est diminuée.

\section{- Relations entre potentiel de base et croissance}

Les courbes des figures $9(\mathrm{~B}, \mathrm{C}$ et $\mathrm{D})$ et $10(\mathrm{~B}, \mathrm{C}$ et $\mathrm{D})$ nous permettent de comparer l'évolution de la croissance et du potentiel de base au cours des traitements. L'arrosage de type $6 \times$ qui favorise un très bon allongement des axes chez les deux espèces maintient un potentiel de base supérieur à -7 bars chez le poirier-pays, mais pouvant atteindre -10 bars chez le courbaril. L'arrosage tri-hebdomadaire permet une remontée du potentiel vers -6 bars pour les deux espèces. Pour ce traitement, seul le courbaril manifeste une croissance notable. 


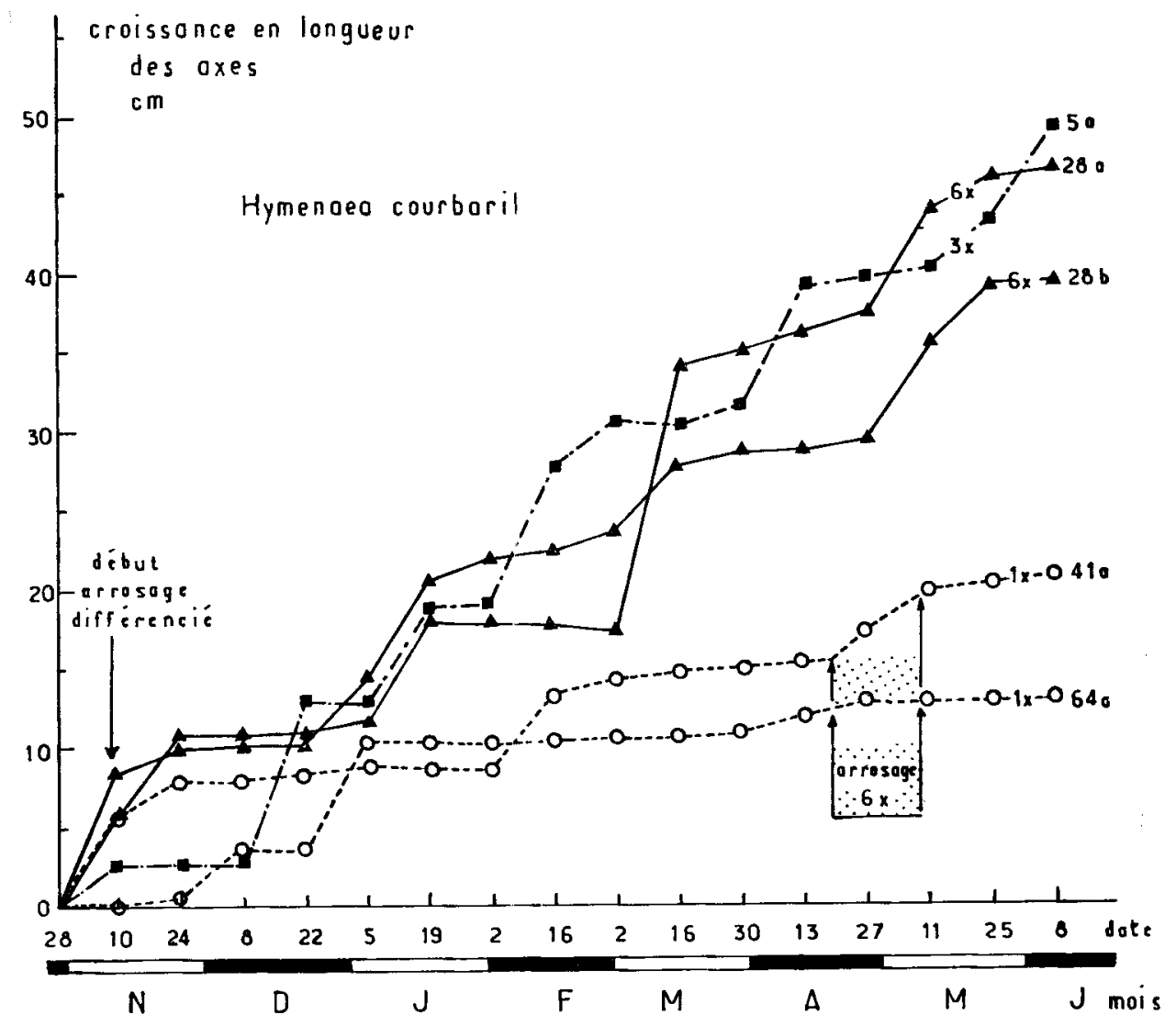

FIG. 8

Croissance individuelle de quelques axes de plants de Hymenaca courbaril dans le dispositif de Duclos.

Growth of sone individual axes of plants of Hymenaea courbaril at Duclos experimental lay-out. 


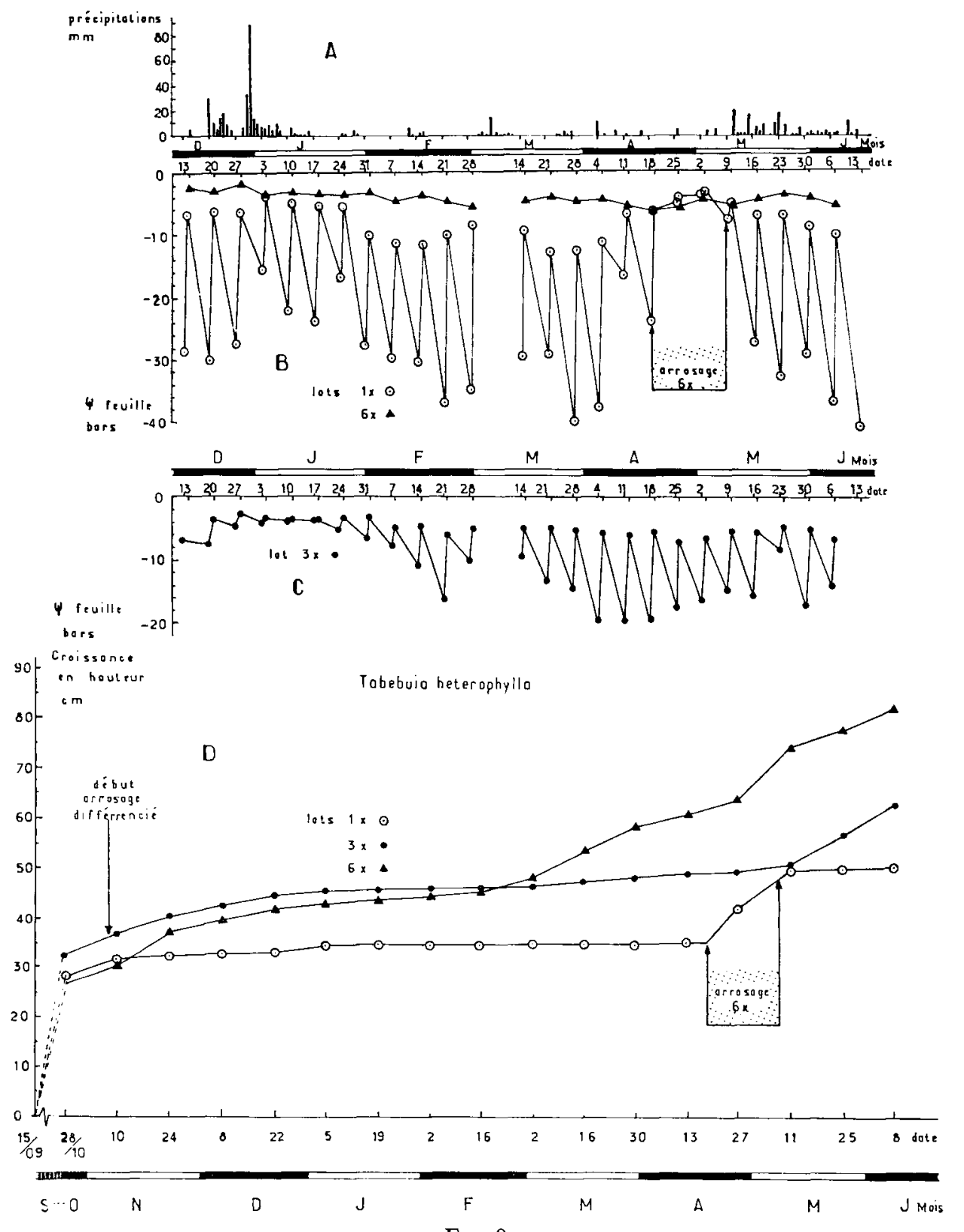

FIG. 9

Evolution du potentiel de base ( $B$ et $C$ ) et de la croissance $(D)$ des plants en fonction des traitements chez. Tabebuia heterophylla dans le dispositif de Blanchet. Potentiels de base les plus élevés et les plus bas atteints chaque semaine pour : les plants arrosés une fois $(1 x)$ et 3 fois $(3 x)$ par semaine.

$P b$ les plus élevés obtenus chaque semaine pour les plants arrosés 6 fois par semaine $(6 x)$. Précipitations enregistrées à Guillard (Basse-Terre) (A).

Evolution of predawn potential $(P b)(B$ and $C)$ and growth $(D)$ in relation with watering regimes in Tabebuia heterophylla in the experimental lay-out at Blanchet. Highest and lowest $\mathrm{Pb}$ obtained every week for : plants watered once (1x) and three times (3x) per week.

Highest Pb obtained every week for plants watered 6 times a week (6x). Rainfalls recorded at Guillard (Basse-Terre) (A). 

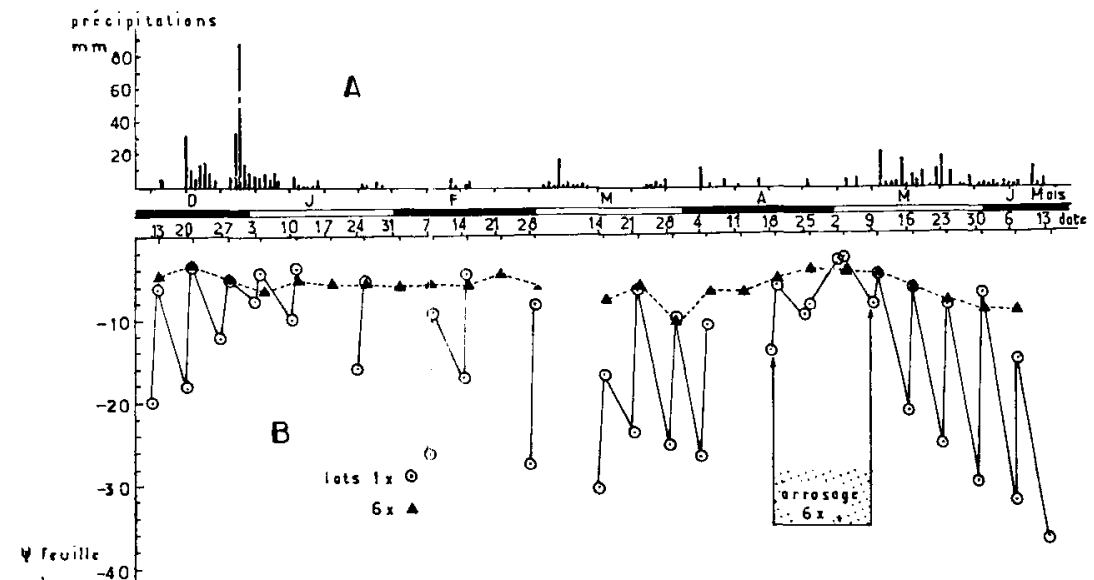

bers -40 P

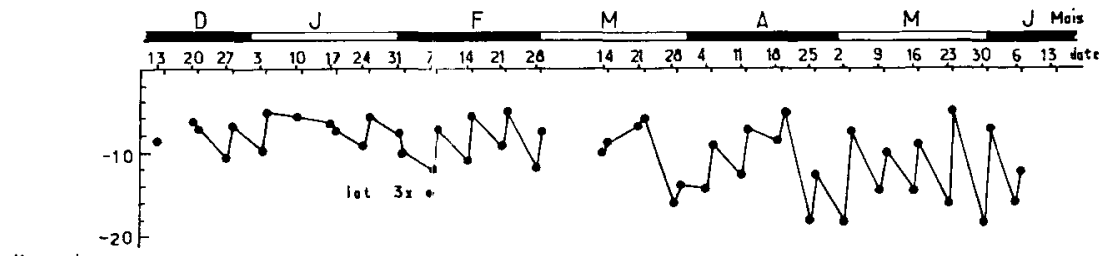

4 reville

bors

croissante

en longueur des axes

$\mathrm{mm}$

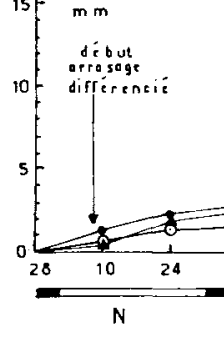

C

Hymenoeo courboril 
Le rythme de 1 arrosage par semaine bloque la croissance chez les deux espèces. Durant cette période, les valeurs de $\mathrm{Pb}$ restent faibles et n'atteignent qu'exceptionnellement -6 bars pour le courbaril et le poirier-pays. On note à ces moments une très faible croissance chez le courbaril uniquement. Après un réarrosage de type $6 \times$, la croissance reprend brutalement dans le lot $1 \times$. Ceci se traduit au niveau du potentiel hydrique par une remontée brutale des valeurs minimales et maximales du $\mathrm{Pb}(-8$ à -3 bars pour le courbaril ; -6 à -4 bars pour le poirierpays). Il y a donc une zone de valeurs de $\mathrm{Pb}$ en-deçà de laquelle la croissance en longueur est possible. Ces premiers résultats, qu'il conviendra de préciser, permettent de penser que ces valeurs limites sont voisines de -6 bars pour le poirier-pays et - 7 bars pour le courbaril. Nous ne pouvons cependant apporter une plus grande précision sur ces niveaux d'arrêt de la croissance car l'intervalle de temps choisi entre deux mesures de croissance successives (15 jours) recouvre 2 arrosages qui peuvent amener le même plant à des potentiels sensiblement différents. Les potentiels mesurés par plant correspondent aux réactions à un seul arrosage, ceci afin d'éviter un trop important prélèvement de feuilles par individu. Ce potentiel n'est pas forcément celui autorisant la croissance. A Blanchet, pour le courbaril, on constate une croissance pour des potentiels inférieurs. Il s'agit en fait de la fin de l'expression de la croissance induite par l'arrosage. La notion d'arrêt de croissance à partir d'un certain potentiel de base est connue chez d'autres espèces de feuillus tels le chêne et le frêne où la croissance s'arrête pour un Pb de -11 bars (Aussenac \& LEVY, 1983).

\subsection{Formation de matière sèche en fonction des traitements}

La formation de matière sèche en fonction du traitement est résumée dans le tableau 4. Les pesées portent sur l'expérience de la pépinière de Duclos et donnent des résultats particulièrement nets. Ainsi le gain de poids sec n'est pas significativement différent suivant que l'on arrose 3 fois ou 6 fois par semaine.

Seul l'arrosage $1 \times$ provoque une diminution significative du poids sec. On notera d'ailleurs que le poids sec des feuilles est sensiblement moins affecté que celui des autres organes chez le courbaril. L'importante défoliaison qui s'est produite dans le lot sec du poirier-pays ne nous a pas permis de comparer les poids secs de ces organes. Les données fournies pour les feuilles des lots $6 \times$ et $3 \times$ sont, à cause d'un début de défoliaison, obtenues par défaut. Elles font apparaître cependant une masse foliaire sensiblement plus importante pour le poirier-pays que pour le courbaril.

Si l'on considère que dans la pépinière de Duclos les plants sont à un état hydrique similaire à ceux de Blanchet, on peut conclure que les valeurs atteintes par le $\mathrm{Pb}$ dans les lots $3 \times$ restent presque toujours compatibles avec l'activité photosynthétique normale, ce qui n'est pas le cas toujours dans le lot sec où ces activités paraissent fortement réduites.

L'évolution du rapport poids sec tiges / poids sec racines (cf. tab1. 5) indique que pour le poirier-pays, le système aérien (tiges) reste comparativement moins affecté que le système souterrain, lors du dessèchement. Par contre, chez le courbaril, les deux systèmes évoluent simultanément. Ceci pourrait signifier que le ralentissement de la croissance racinaire n'affecte pas la. croissance des tiges du poirier-pays. 


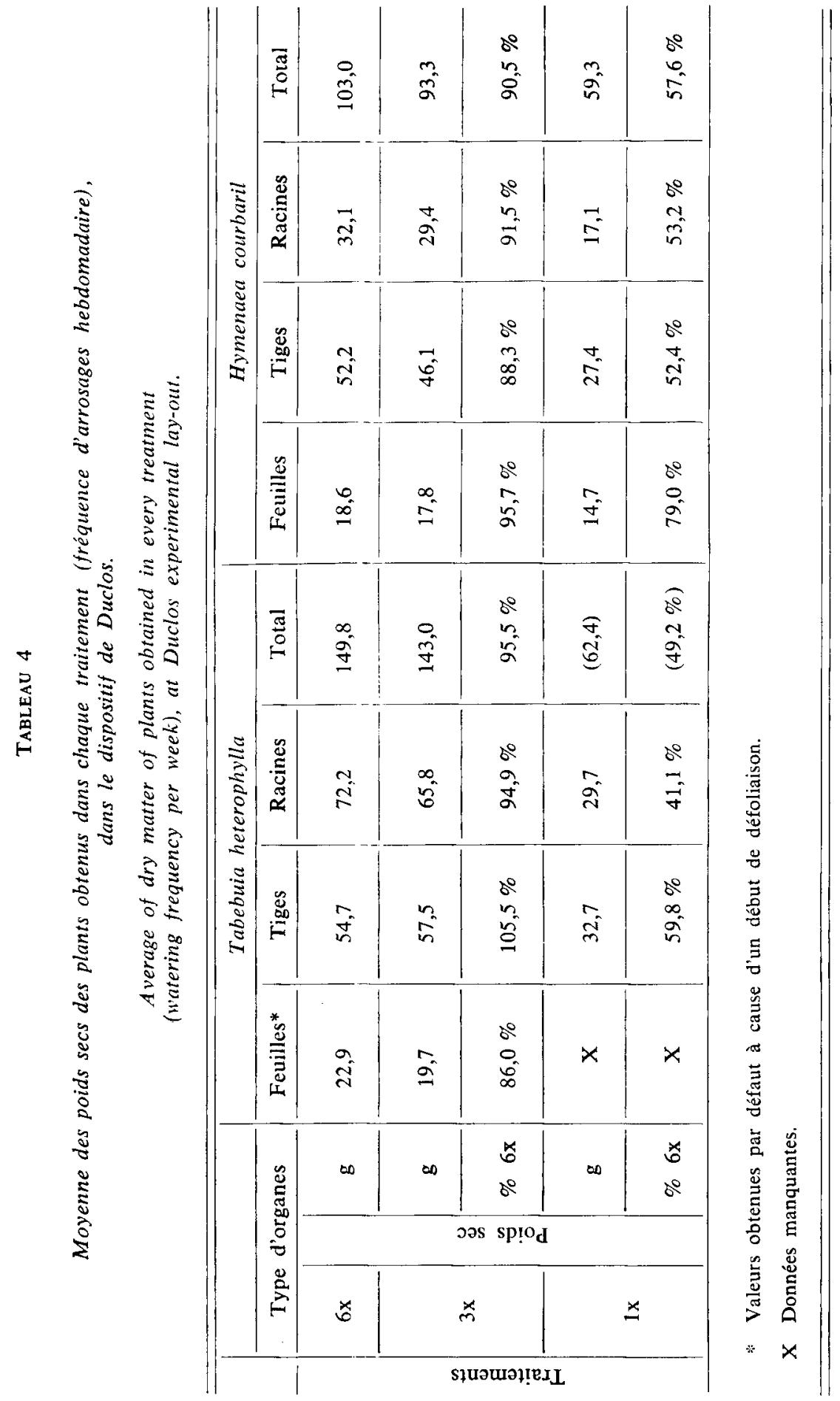


TABleau 5

Valeur du rapport Poids sec tiges/Poids sec racines des plants dans les différents traitements (fréquence d'arrosages par semaine).

Ratio dry matter of stems/dry matter of roots of the plants in the different treatments (watering frequency per week).

\begin{tabular}{c|c|c}
\hline \hline \multirow{2}{*}{ Traitement } & \multicolumn{2}{|c}{ Espèces } \\
\cline { 2 - 3 } & T. heterophylla & Hymenaea courbaril \\
\hline $6 \mathrm{x}$ & 0,76 & 1,62 \\
$3 \mathrm{x}$ & 0,87 & 1,57 \\
$1 \mathrm{x}$ & 1,10 & 1,60 \\
\hline
\end{tabular}

\section{Conclusion}

On peut noter au terme de cette étude que ces plants peuvent tolérer un dessèchement important du sol qui se traduit par un potentiel hydrique très faible de l'ordre de -50 bars, et retrouver rapidement après un réarrosage un potentiel élevé favorable à la croissance. D'une manière pratique, on voit qu’une fréquence de 3 arrosages par semaine suffit à maintenir un très bon développement des plants en pépinière pour ces espèces qui tirent un faible avantage d'une suralimentation hydrique.

Cette aptitude de résistance à la sécheresse est doublée chez ces espèces par un phénomène d'évitement de la sécheresse. Le poirier-pays, au cours d'un déficit hydrique prolongé, perd ses feuilles. De même le courbaril perd ses feuilles en début de saison sèche; toutefois, son rythme de défoliaison est moins souple car étroitement lié à la photo-période (Huc, en préparation). Du point de vue bio-climatique, ceci constitue un net avantage pour le poirier-pays qui peut à tout moment réduire sa surface transpirante en fonction des conditions d'évapotranspiration du moment, phénomène important pour cette espèce qui, si on tient compte du caractère significatif du potentiel critique $\left(\mathrm{Pb}_{0}\right)$, voit ses aptitudes de photosynthèse et de transpiration se réduire pour un potentiel hydrique plus élevé que celui observé pour le courbaril. Ceci dénote le caractère «opportuniste» du poirier-pays, espèce pionnière de la zone mésophile et de la forêt xérophile.

\section{Remerciements}

Ce travail a pu être réalisé grâce à la collaboration de l'Office National des Forêts. Que soient ici sincèrement remerciés :

- M. F. Wencelius, Directeur de l'O.N.F. de la région Guadeloupe,

- MM. C. Raynaud et Z. Cimon qui nous ont offert toutes les facilités dans les pépinières respectives de Pointe-à-Pître et de Blanchet,

- le personnel technique de l'O.N.F., et plus particulièrement $M^{\text {me }}$ M.R. Haral, ainsi que MM. Boucher et Clarence.

Nous tenons également à remercier tout particulièrement ici :

- M. Ch. Valancogne, Directeur de la Station de Bioclimatologie de l'I.N.R.A., Antilles-Guyane, qui nous a offert l'appui technique de son équipe, 
- les techniciens de la Station de Bioclimatologie qui ont participé à des titres divers à ce travail,

- MM. P. Artis et R. Boula pour l'étude des sols,

- MM. S. Leinster, T. Mesinele, D. Taupe et P. Vinglasson qui ont participé à l'installation et au maintien des dispositifs ainsi qu'à la récolte des données de croissance et de poids sec.

\author{
Summary \\ First experimental data on behaviour of Tabebuia heterophylla (DC) Britton \\ and Hymenaea courbaril L. in relation to drought
}

Two tree species of the lowland forest of tropical America (Antilles), Tabebuia heterophylla («poirier-pays》 or « white cedar») and Hymenaea courbaril (« courbaril» or «locust», believed to be adapted for reforestation in less humid areas. The drought resistance of these species is studied.

The evolution of the water status in the plant is studied during a six months of experimentation using the pressure bomb technique. Characteristic values of water potential, predawn potential $(\mathrm{Pb})$ and minimum potential $(\mathrm{Pm})$, are given. Diurnal variation of predawn potential indicates that white cedar is more sparing of its water than locust. This seems to be due to its more developped root system which produces drying conditions in the soil within a short period of time. This is evident by a rapid decrease of predawn potential of this species under short watering frequency (watering once per week).

During the drying phase the evolution of the difference $\mathrm{Pm}-\mathrm{Pb}$ indicates maximal stomatal closure at a predawn potential which is higher for white cedar ( -25 bars) than locust (-32 bars).

Data from longitudinal growth of axes indicate that the growth is stopped for both species at high values of $\mathrm{Pb}$ near -6 bars for white cedar and -7 bars for locust.

For both species, only watering once a week significantly reduces dry matter formation and growth in the plants.

\title{
Références bibliographiques
}

Aussenac G., Granier A., 1978. Quelques résultats de cinétique journalière du potentiel de sève chez les arbres forestiers. Ann. Sci. for., 35 (1), 19-32.

Aussenac G., Levy G., 1983. Influence du dessèchement du sol sur le comportement hydrique et la croissance du chêne pédonculé (Quercus pedunculata Ehrl.) et du frêne (Fraxinus excelsior L.) cultivés en cases de végétation. Ann. Sci. for., 40 (3), 251-264.

Huc R. (en préparation). Le courbaril (Hymenaea courbaril L.). Caractéristiques botaniques et biologiques générales, synthèse bibliographique.

LASSERRE G., 1961. La Guadeloupe. I. La nature et les hommes. Union Française d'Impression, Bordeaux, 448 p.

Reich P.B., Borchert R., 1982. Phenology and ecophysiology of the tropical tree, Tabebuia neochrysantha (Bignoniaceae), Ecology, 63 (2), 294-299.

Scholander P.F., Hammel H.T., Bradstreet E.D., Hemmingsen E.A., 1965. Sap pressure in vascular plants, Science, 148, 339-345.

Waring R.H., Cleary B.D., 1967. Plant moisture stress : evaluation by pressure bomb, Science, 155, 1248-i254. 